\title{
Perfil epidemiológico da morbi-mortalidade masculina
}

\author{
Epidemiological profile of men: \\ morbidity and mortality
}

Ruy Laurenti 1

Maria Helena Prado de Mello Jorge 1

Sabina Léa Davidson Gotlieb 1

1 Departamento de Epidemiologia da Faculdade de Saúde Pública da Universidade de São Paulo.

Av. Dr. Arnaldo 715, 01246-904, São Paulo SP. laurenti@usp.br
Abstract Differences between aspects of women and men health are pointed out and questions about biologic (sex) and behavioral (gender) factors are commented. The higher male mortality and their lower life expectancy, as well as the aging process of the population are discussed. The actual Brazilian male mortality pattern is similar to other countries. The male death rates are 50\% higher than the female ones. Considering the age groups, the higher sex ratio occurred in the age group 20 to 39 years ( 3 male deaths: 1 female death). Observing the distributions of deaths according to the underlying causes, for both sexes, call the attention deaths by diseases of the circulatory system, in first place, and then, external causes (injury, poisoning and certain other consequences). Taking in account morbidity, measured by hospital discharges (excluding all related to childbirth/deliverance), there is an equilibrium between the numbers of male and female hospitalizations. There is similarity among the male and the female distributions of hospital discharges according to the reason of hospitalization, besides the case of external causes and mental and behavioral disorders, with sex ratio of 2.3 and 1.9 male hospital discharges to each female discharge. Aids and alcoholism are discussed as examples of important disorders in the male health status

Key words Epidemiology, Male mortality, Male morbidity, Male health, Sex/Gender
Resumo São abordados aspectos das diferenças entre a saúde do homem e da mulher, enfocando questões ligadas a fatores biológicos (sexo) e comportamentais (gênero). A sobremortalidade masculina e a resultante menor esperança de vida estão apresentadas; são discutidos o envelhecimento populacional e suas conseqüências, do ponto de vista da saúde, sendo estas mais intensas no homem. O panorama atual no Brasil segue o padrão observado em outros países. Os coeficientes de mortalidade masculina são cerca de 50\% maiores e, considerando as idades, a maior razão de sexo acontece no grupo etário de 20 a 39 anos (3 mortes masculinas para cada feminina). Na distribuição segundo causas, sobressaem mortes por doenças do aparelho circulatório seguidas por aquelas relativas a acidentes e violências. Na morbidade, medida pelas internações (excluídas aquelas por parto), há um equilíbrio entre o número de hospitalizações masculinas e femininas. As distribuições das altas segundo os motivos de internação, para ambos os sexos, apresentam semelhanças, com exceção das relativas a causas externas e aos transtornos mentais e comportamentais, respectivamente, com razões iguais a 2,3 e 1,9 internações masculinas para cada feminina. São estudados ainda a Aids e o alcoolismo, como exemplos de importantes agravos na saúde masculina. Palavras-chave Epidemiologia, Mortalidade masculina, Morbidade masculina, Saúde masculina, Sexo e gênero 


\section{Diferenciais na saúde do homem e da mulher}

Diferenciais nas características de saúde entre os sexos são bem conhecidos, sendo que a questão dos determinantes biológicos e daqueles ligados ao gênero foi amplamente comentada para os países da Região das Américas (Laurenti et al., 1998).

A maioria dos indicadores tradicionais de saúde mostra, com clareza, a existência desse diferencial, sendo maior a mortalidade masculina em praticamente todas as idades e para quase a totalidade das causas; também as esperanças de vida ao nascer e em outras idades são sempre menores entre os homens. No Brasil, essas diferenças, que eram de aproximadamente cinco anos, durante as décadas anteriores a 1980, elevaram-se nas décadas seguintes, sendo que, para 2001, as mulheres tinham maior sobrevida de oito anos, em relação à esperança de vida masculina (respectivamente, 73 e 65 anos). Este fato é mais intenso na região Sudeste, especialmente no Rio de Janeiro, onde a diferença atinge 12 anos (respectivamente 74 e 62 anos) (Ripsa, 2004). Em países desenvolvidos, a diferença é de aproximadamente cinco anos, como nos Estados Unidos e Canadá (OPS, 2003).

Ainda que os indicadores de mortalidade evidenciem situações desfavoráveis para os homens, os referentes à morbidade, medidos pela demanda aos serviços e por inquéritos populacionais, destacam, de uma maneira geral, maior freqüência para as mulheres.

Não há dúvida de que existem alguns determinantes biológicos para as heterogeneidades encontradas, como por exemplo, a grande sobremortalidade masculina por complicações da aterosclerose coronária; entretanto, nas idades mais velhas, após a menopausa, com a perda da proteção hormonal, há aumento acentuado da mortalidade feminina por essas causas, diminuindo a preponderância dos homens. De outra parte, há agravos à saúde, ligados a comportamentos específicos do homem e da mulher, que dependem de fatores culturais e, de uma maneira geral, sociais.

Durante algum tempo, os termos sexo e gênero eram utilizados indiscriminadamente; na literatura mais recente, entretanto, o termo sexo reserva-se, preferentemente, às características biológicas predeterminadas, relativamente invariáveis, do homem e da mulher, enquanto que gênero é utilizado para assinalar as características socialmente construídas que constituem a definição do masculino e do feminino, em diferentes culturas (OPS, 1993).

Hartigan (1998) comenta que, na América Latina e no Caribe, é comum aceitar-se que gênero é sinônimo de mulher. Há de ser ressaltado, entretanto, que gênero deve ser entendido como a rede de traços de personalidade, atitudes, sentimentos, valores, condutas e atividade que, através de um processo de construção social, diferencia os homens das mulheres, como muito bem explicita a publicação da OPS Gênero, mujer $y$ salud (OMS, 1995).

Na publicação da OPS, Perfil epidemiológico de la salud de la mujer en la región de las Américas (OPS, 1990a) são feitos os seguintes comentários: "sexo", masculino ou feminino, é uma condição biológica determinada pela natureza e, basicamente, pelas leis da genética. Fazendo abstração dos desvios cromossômicos que dão lugar a fenótipos e genótipos que são exceçães naturais, o sexo tem uma base biológica que determina uma anatomia corporal e uma fisiologia determinada. Por outro lado, o que se denomina "gênero" (homem ou mulher) é, ao invés, uma construção social a partir da diferença entre os sexos que, portanto, varia historicamente e está sujeita a mudanças por intervenções de políticas na ordem social, econômica, jurídica e política. O ser homem ou mulher e as relações que se estabelecem entre os mesmos obedecem a papéis definidos pela sociedade em determinados períodos de evolução histórica da humanidade.

A sobremortalidade dos homens foi descrita há séculos e, aparentemente, sua primeira mensuração foi feita por John Graunt, em 1662, que, na publicação Natural and political observations made upon the bills of mortality, fez comentários a respeito (MacMahon \& Pugh, 1970).

A menor sobrevida masculina foi - ou ainda é - quase sempre aceita sem muita discussão e vista como resultado somente de fatores biológicos. Acresce-se a isso o fato de ser continuamente mencionado que nascem e, em todas as idades, morrem mais homens, obtendo-se, assim, o equilíbrio entre os sexos. É inegável a existência do fator biológico, porém, o enfoque de gênero não pode deixar de ser considerado quando se deseja caracterizar e analisar a saúde do homem e da mulher.

Ainda que as análises de mortalidade ou de morbidade, sob enfoque de gênero, nas últimas décadas, tenham sido quase que exclusivamente dirigidas para o sexo feminino, há algumas publicações que abordam ou, pelo menos, tangenciam essa questão em relação ao sexo masculi- 
no. Há mais de quarenta anos, Madigan (1957) publicou o interessante trabalho Are mortality differentials biologically caused?, no qual questionava a razão de o homem não ter aproveitado, da mesma forma que a mulher, as melhorias das condições gerais de vida que as primeiras décadas do século 20 trouxeram, bem como indagava sobre quais seriam as probabilidades de que a vida masculina fosse prolongada até igualar-se à das mulheres. Não deixa de ser interessante a opinião do autor de que seria mais fácil atuar nos fatores biológicos do que nos comportamentais ou sociais, principalmente, levando em consideração que o estado atual da engenharia genética, em grande avanço, possibilita alterar o curso de algumas doenças ou, até mesmo, seu desaparecimento, ao intervir em determinados genes.

\section{O envelhecimento da população}

O aumento considerável do número de idosos na população é observado tanto nos países desenvolvidos quanto naqueles em desenvolvimento, como é o caso do Brasil (Torrey et al., 1987); e essa população de idosos, por sua vez, está se tornando mais "velha". De fato, o número de habitantes com idade mínima de 80 anos, às vezes chamados "os velhos mais velhos", está crescendo rapidamente em muitos países (OMS, 1995a). Nos Estados Unidos, a população de homens brancos de 85 e mais anos representava $0,32 \%$ e $0,74 \%$ dos homens, respectivamente em 1950 e 1990, devendo-se lembrar que era inferior aos valores femininos (NCHS, 1993).

No Brasil, entre os homens, as proporções de idosos de 60 anos e mais eram iguais a 5,8\%, $6,8 \%, 7,3 \%$ e $7,8 \%$, respectivamente, em 1980 , 1991, 1996 e 2001, e as de 80 anos e mais eram, respectivamente, $0,4 \%, 0,6 \%, 0,8 \%$ e $0,9 \%$.

Do ponto de vista da saúde, surgem novos problemas ligados ao envelhecimento, destacando-se as conseqüências das doenças crônicas. Assim, o setor saúde tem de estar preparado para enfrentar o incremento do número de casos, em um grupo populacional de características específicas. Em 2002, do total de internações masculinas em hospitais do Sistema Único de Saúde (SUS) ou com eles conveniados, 4,5\%, $12,4 \%$ e $21,1 \%$ foram por neoplasias malignas, doenças dos aparelhos circulatório e respiratório, respectivamente, enquanto que para os de 60 e mais anos, os valores foram 7,3\%, 29,4\% e $20,1 \%$. Para os maiores de 80 anos, as propor- ções chegaram a 4,8\%, 30,5\% e 25,1\%, para as respectivas causas.

Estudos longitudinais, mostrando a relação entre a crescente longevidade e as deficiências e incapacidades, indicam que a população está vivendo mais tempo com um grau crescente desses problemas (Verbrugge, 1989). Igualmente, Van Nostrand et al. (1993) afirmam que, por não ter mudado a idade da incidência ou do aparecimento das principais doenças crônicas, há uma prevalência maior de deficiências e incapacidades nas idades avançadas. Portanto, a reabilitação é uma questão fundamental na assistência oferecida aos idosos. Ainda que esse aspecto seja comum a ambos os sexos e, mesmo, numericamente superior nas mulheres, dada a sua maior sobrevida, não se pode deixar de chamar a atenção para o mesmo quando se aborda a saúde do homem.

\section{Determinantes da saúde do homem}

As transformações nos padrões de saúde/doença constituem-se em uma das características do século 20, estando associadas às mudanças na estrutura etária populacional. Para a análise da saúde do homem, no Brasil, no presente momento, é preciso invocar as transições demográfica e epidemiológica, com o conseqüente envelhecimento populacional e alterações no panorama das doenças.

No que tange ao homem, verifica-se que a diminuição da sua mortalidade foi mais lenta e sempre menor do que a observada no sexo feminino; como decorrência, atualmente, a vida média masculina é comparativamente menor em todas as regiões do Brasil. É de se destacar, e de grande interesse para a saúde, que a proporção de idosos é menor entre os homens. No País, em 2001, 7,8\% do total de homens estavam na faixa etária igual a 60 e mais anos e $0,9 \%$ tinham no mínimo 80 anos; para as mulheres esses valores foram maiores, respectivamente, iguais a $9,3 \%$ e $1,3 \%$.

A transição epidemiológica, após a demográfica, caracteriza-se, de um lado, pelo decréscimo da mortalidade infantil, em decorrência da queda acentuada das doenças infecciosas e, do outro, pela elevação dos coeficientes por doenças não-infecciosas, particularmente, cardiovasculares, neoplasias malignas e diabetes (Laurenti, 1990).

O Brasil, do ponto de vista da saúde de sua população, apresenta o paradoxo de conviver, 
ao mesmo tempo, com situação predominante nos países desenvolvidos (doenças não-infecciosas) e com doenças já vencidas há décadas, como várias doenças infecciosas, além, ainda, de altos valores da mortalidade infantil, em algumas áreas.

Bobadilha et al. (1993) discutem as transformações ocorridas e que resultaram de complexa interação de vários fatores com atuação marcante na saúde da população humana. Alguns desses influenciaram muito mais a saúde do homem do que da mulher, o que é válido para o homem brasileiro e suas características atuais de saúde.

Waldman (2000) comenta que essas transformações, positivas em muitos pontos, trouxeram também novos desafios cujas soluções ainda aguardam equacionamento adequado. São exemplos desse desafio: o envelhecimento da população, ampliação da morbi-mortalidade decorrente da violência, da exposição aos riscos ambientais, de causas associadas às mudanças de estilo de vida e ao comportamento e as alterações das características das doenças infecciosas.

Várias doenças acometem mais os homens, traduzindo-se por maior mortalidade desse sexo. Essa é uma questão que precisa ser levada em conta quando se pensa em saúde da família e, por extensão, em uma comunidade saudável. De fato, em geral, há programas de saúde que contemplam a criança, o adolescente, a mulher e o idoso, não existindo, porém, com exceção dos programas de saúde do trabalhador, aqueles voltados especificamente para o homem adulto. Não que seja desejável existir um programa somente dirigido a ele mas, no programa de saúde da família ou do sub-programa de saúde do adulto, devem ser levantadas algumas questões específicas para o homem, como, por exemplo, ações educativas em relação às violências e aos cânceres de próstata e de pulmão.

Em relação ao câncer de próstata, a mortalidade vem aumentando em várias regiões das Américas, atingindo, algumas vezes, o terceiro e mesmo o segundo lugar entre os cânceres mais freqüentes no sexo masculino. Entretanto, aparentemente, não existe tanto interesse das autoridades sanitárias em estabelecer atividades educativas voltadas para essa causa e, quando existem, os homens não são sensíveis a elas. Esse comportamento é diferente daquele verificado entre as mulheres com relação à adesão a programas para a detecção precoce dos cânceres de mama e do colo de útero. A análise sob o enfoque de gênero muito contribuiria para o enten- dimento dessas situações. Para o câncer de pulmão é fundamental existirem programas educativos voltados para a diminuição do hábito de fumar cigarros.

Um melhor conhecimento do que está ocorrendo em várias regiões, em relação ao binômio homem-violência, traria contribuição importante para a inserção de alguns aspectos específicos nos programas educativos voltados para a sua prevenção. Cabe ao setor saúde o alerta a respeito desse problema e até mesmo a coordenação dos programas de prevenção às violências, tendo em vista que é sobre esse setor que recaem os elevados custos representados pelo atendimento às vítimas e suas seqüelas.

A maior presença feminina observada na demanda aos serviços de saúde, muito provavelmente, está associada a fatores culturais ou sociais. $\mathrm{O}$ fato de caber à mulher, em geral, acompanhar crianças, adolescentes e idosos a esses serviços de saúde, além de, em determinado período de sua vida, freqüentar o pré-natal, faz com que ela se torne, provavelmente, mais predisposta à utilização desses serviços. Essa alta morbidade de demanda, diante da baixa mortalidade, que pode parecer paradoxal, tem sido descrita, também, em inquéritos de morbidade, em particular nos Estados Unidos, onde foi mostrado que as mulheres apresentavam maior incidência de episódios agudos de doenças, maior tempo de restrição de atividades por doenças, maior número de internações hospitalares, de visitas a médicos, bem como, maior número de dias que permaneceram acamadas por doença (Adams \& Benson, 1990).

\section{Mortalidade masculina}

\section{Aspectos gerais}

John Graunt, em 1662, apresentou pela primeira vez dados sobre mortalidade e mostrava o que ocorria com os homens e com as mulheres, na população que morria em Londres. Desde então, foi o primeiro a descrever a sobremortalidade dos homens. À medida que outras cidades, regiões ou países passaram a elaborar suas estatísticas de mortalidade, o mesmo fato foi constatado, tornando-se, até hoje, uma constante.

A maior mortalidade masculina é observada não somente quanto aos números absolutos e respectivos coeficientes, mas também quanto a causas. Excetuando-se as doenças próprias ou 
específicas do sexo, para praticamente todas as causas, são maiores os coeficientes masculinos, sendo tal fato observado em todas as idades.

Murray et al. (1992) calcularam a probabilidade de morte de adultos em ambos os sexos e em diferentes grupos etários. Foi feito o cálculo da probabilidade de morte $-{ }_{45} \mathrm{Q}_{15}$ - sendo que, nesta notação, o primeiro número indica o número de anos no intervalo e o segundo refere a idade na qual o intervalo se inicia. Dessa maneira, ${ }_{45} \mathrm{Q}_{15}$ é a probabilidade de morrer entre $15 \mathrm{e}$ 60 anos de idade, medida em porcentagem; de uma maneira mais didática, representa a porcentagem daqueles com 15 anos que irão morrer antes de completar 60 anos.

Os autores descrevem que, para os países em desenvolvimento, no seu conjunto, $\mathrm{a}_{45} \mathrm{Q}_{15}$ é igual a $25 \%$ para os homens e $22 \%$ para as mulheres. Isto indica que, aproximadamente, um dentre quatro homens de 15 anos morrerá antes de completar 60 anos, sendo essa proporção menor para as mulheres. Ao comparar com um país desenvolvido - no caso, o Japão - os autores mostram valores bem menores, iguais a $11 \% \mathrm{e}$ $6 \%$, respectivamente, para homens e mulheres.

O cálculo deste indicador pode ser feito para doenças específicas. Considerando ${ }_{45} \mathrm{Q}_{15}$ específicas para algumas causas, para um conjunto de 56 países com registro vital completo, bem como com boa declaração da causa de morte, encontraram valores médios iguais a $0,79 \%$ e $0,49 \%$, respectivamente, para homens e mulheres, no caso das doenças transmissíveis e aquelas ligadas à reprodução, isto é, 0,79\% dos homens e $0,49 \%$ das mulheres com 15 anos não atingirão ou morrerão antes de completar 60 anos, por estes agravos. Para as doenças não transmissíveis, os valores encontrados foram, respectivamente, $12,92 \%$ e $8,04 \%$ e, para causas externas, $4,07 \%$ e $1,11 \%$. Considerando todas as causas selecionadas, a ${ }_{45} \mathrm{Q}_{15}$ é maior para os homens, sendo a razão homem/mulher de 1,61, 1,60 e 3,67, respectivamente, para as doenças transmissíveis e aquelas ligadas à reprodução, as não-transmissíveis e as causas externas. A observação da maior mortalidade masculina leva a comentar que é difícil interpretá-la justificando-a como devida ao sexo (variável biológica), parecendo muito mais pertinente ser atribuída a fatores sociais e comportamentais (variável gênero). Isso fica claro não somente para as causas externas mas também para várias causas naturais: câncer de pulmão, doença pulmonar obstrutiva crônica, cirrose hepática, entre outras.

\section{Panorama no Brasil de hoje}

O padrão de mortalidade masculina, no Brasil, segue aquele observado em outros países, desenvolvidos ou em desenvolvimento. Os óbitos masculinos segundo grupos etários, analisados em série histórica de 1980 a 2001 (Tabela 1), mostram dois aspectos importantes: em primeiro lugar, há uma grande redução da participação do grupo de menores de cinco anos, de $27,8 \%$ para $7,3 \%$, respectivamente, em 1980 e 2001; o outro ponto é o aumento da mortalidade proporcional entre os idosos, isto é, de indivíduos de 60 anos e mais, de $28 \%$ para $35 \%$ e de $7,7 \%$ para $15,2 \%$, nos grupos 60 a 79 anos e 80 anos e mais, respectivamente, nos dois anos. É preciso citar que o mesmo ocorreu entre as

Tabela 1

Número e proporção (\%) de óbitos do sexo masculino segundo idade. Brasil, 1980/2001.

\begin{tabular}{|c|c|c|c|c|c|c|c|c|}
\hline \multirow[t]{2}{*}{ Idade } & \multicolumn{2}{|c|}{1980} & \multicolumn{2}{|c|}{1991} & \multicolumn{2}{|c|}{1996} & \multicolumn{2}{|c|}{2001} \\
\hline & No & $\% *$ & No & $\%^{*}$ & No & $\%{ }^{*}$ & No & $\%{ }^{*}$ \\
\hline 0 a 4 & 118.973 & 27,8 & 57.091 & 12,2 & 49.011 & 9,4 & 40.429 & 7,3 \\
\hline 5 a 9 & 5.057 & 1,2 & 3.883 & 0,8 & 3.556 & 0,7 & 3.016 & 0,5 \\
\hline 10 a 19 & 13.764 & 3,2 & 16.273 & 3,5 & 18.315 & 3,5 & 19.063 & 3,4 \\
\hline 20 a 39 & 52.494 & 12,3 & 77.589 & 16,5 & 87.787 & 16,8 & 86.258 & 15,5 \\
\hline 40 a 59 & 84.678 & 19,8 & 102.953 & 22,0 & 117.334 & 22,4 & 128.686 & 23,1 \\
\hline 60 a 79 & 119.537 & 28,0 & 154.056 & 32,9 & 177.061 & 33,8 & 195.091 & 35,0 \\
\hline 80 e mais & 32.810 & 7,7 & 56.532 & 12,1 & 70.153 & 13,4 & 84.612 & 15,2 \\
\hline Ignorada & 3.848 & * & 6.528 & * & 6.851 & * & 3.488 & * \\
\hline Total & 431.161 & 100,0 & 474.905 & 100,0 & 530.068 & 100,0 & 560.643 & 100,0 \\
\hline
\end{tabular}

* \% calculada sobre o total de casos com informação conhecida.

Fonte: www.datasus.gov.br (dados brutos). 
mulheres; entretanto, tanto a diminuição porcentual nas primeiras idades como o aumento nos idosos, foram mais acentuados.

Interessante é o fato de ter aumentado, ainda que ligeiramente, a proporção masculina na mortalidade geral (de $57,5 \%$ para $58,4 \%$ ). Em ambos os sexos, houve redução dos coeficientes de mortalidade, porém, essa redução foi um pouco mais acentuada entre as mulheres (superior a $10 \%)$ do que entre os homens $(6,8 \%$ de queda). Os coeficientes passaram de 7,3 para 6,8 por mil homens e, os femininos, de 5,3 para 4,6 por mil mulheres. Por outro lado, como esperado, os coeficientes masculinos foram maiores que os femininos, em cerca de $50 \%$, nos quatro anos.

Ao serem analisados os coeficientes específicos por grupos etários, constata-se que a razão de sexo entre eles atinge valores altos, dos 10 aos 39 anos (Tabela 2). Esta situação se deve ao predomínio das causas externas nessas idades, pois somente não se posicionaram, entre as principais, nos idosos (Tabela 3). Nos óbitos de adolescentes (10 a 19 anos) e de adultos jovens (20 a 39 anos), sua presença foi, respectivamente, de 77,8\% (menos de um quarto dos óbitos apresentam outras causas básicas) e 64,8\%.

$\mathrm{Na}$ análise dos óbitos por causas básicas de morte, classificados segundo capítulos da Classificação Internacional de Doenças, 10a Revisão - CID-10 (OMS, 1995), nota-se sobremortalidade masculina, para a grande maioria de causas (Tabela 4). Mais uma vez, chamam a atenção os coeficientes de mortalidade por causas externas (razão de sexo entre os coeficientes igual a 5,5). Dentre essas causas, o tipo de violência mais importante é o homicídio, vindo a

\section{Tabela 2}

Coeficientes de mortalidade (por mil hab.) segundo sexo e idade, Brasil, 2001.

\begin{tabular}{lccc}
\hline Idade & Masculino & Feminino & Razão M/F \\
\hline 0 a 4 & 4,8 & 3,9 & 1,23 \\
5 a 9 & 0,4 & 0,3 & 1,33 \\
10 a 19 & 1,1 & 0,4 & 2,75 \\
20 a 39 & 3,1 & 1,0 & 3,10 \\
40 a 59 & 8,3 & 4,3 & 1,93 \\
60 a 79 & 33,2 & 21,4 & 1,55 \\
80 e mais & 114,4 & 97,7 & 1,17 \\
Total $^{*}$ & 6,6 & 4,6 & 1,43 \\
\hline
\end{tabular}

* Incluindo idade ignorada.

Fonte: www.datasus.gov.br (dados brutos) seguir os acidentes de transporte. Outras causas com acentuada sobremortalidade masculina são os transtornos mentais e comportamentais (razão igual a 4,2), que englobam aqueles devido ao uso de álcool e de outras substâncias psicoativas, significativamente associados ao homem. Seguem-se, as mortes por doenças do aparelho digestivo (razão de sexo igual a 1,9), com destaque para a cirrose hepática, informada ou não com associação ao alcoolismo.

Embora não exista grande diferença nos coeficientes de mortalidade por neoplasias malignas, é preciso destacar que, entre os homens, predominam os cânceres de pulmão, vindo a seguir os de próstata e o de estômago. A análise de séries históricas de mortalidade no Brasil mostra claramente a importância crescente do câncer de próstata que já suplanta a neoplasia maligna de estômago, há alguns anos.

\section{Morbidade masculina}

\section{Aspectos gerais}

John Graunt, nos seus escritos do século 17, comentava o fato de ser menor a freqüência de doenças nos homens, descrevendo que os médicos atendiam duas vezes mais mulheres do que homens, mas que estes morriam mais do que as mulheres (Rothman, 1996).

Na publicação Perfil epidemiológico da saúde masculina na Região das Américas - Uma contribuição para o enfoque de gênero (Laurenti et al., 1998), os autores comentam que a preponderância feminina na morbidade não era observada em algumas doenças de notificação compulsória, nas quais a incidência estava associada ao fator ocupacional, e citam, como exemplos, para o Estado de São Paulo, tétano, leishmaniose tegumentar americana e leptospirose. Acidentes ofídicos e as intoxicações por produtos químicos industriais e pesticidas também são mais freqüentes em homens.

É fato bastante conhecido que, nos inquéritos domiciliares, as mulheres relatam queixas com maior freqüência, bem como estão mais presentes nos serviços ambulatoriais. Em pesquisa realizada em área da Região Metropolitana de São Paulo, em 1989/1990, encontrou-se, em todas as idades, uma proporção de aproximadamente $50 \%$ a mais de mulheres que apresentavam alguma queixa (César et al., 1991).

O projeto multicêntrico - SABE - Saúde, Bem-estar e Envelhecimento (Lebrão \& Lau- 
Tabela 3

Óbitos masculinos (\%) segundo principais causas (capítulos CID 10)*. Brasil, 2001.

\begin{tabular}{lccccc}
\hline Idade (em anos) & 1o Posto & 2o Posto & 3o Posto & 4o Posto & 5o Posto \\
\hline 0 a 9 & Perinatais & An. Congênitas & Infecciosas & D. Ap. Resp. & Externas \\
& $44,2 \%$ & $10,1 \%$ & $8,7 \%$ & $8,4 \%$ & $7,1 \%$ \\
10 a 19 & Externas & Neoplasias & D. Ap. Circ. & D. Ap. Resp. & Infecciosas \\
& $77,8 \%$ & $5,0 \%$ & $3,4 \%$ & $3,2 \%$ & $3,1 \%$ \\
20 a 39 & Externas & Infecciosas & D. Ap. Circ. & D. Ap. Resp. & Neoplasias \\
& $64,8 \%$ & $8,6 \%$ & $7,4 \%$ & $5,1 \%$ & $4,2 \%$ \\
40 a 59 & D. Ap. Circ. & Externas & Neoplasias & D. Ap. Dig. & Infecciosas \\
& $30,0 \%$ & $19,5 \%$ & $16,0 \%$ & $10,5 \%$ & $7,0 \%$ \\
60 a 79 & D. Ap. Circ. & Neoplasias & D. Ap. Resp. & Endócrinas & D. Ap. Dig. \\
& $41,4 \%$ & $20,8 \%$ & $13,1 \%$ & $6,0 \%$ & $5,8 \%$ \\
80 e mais & D. Ap. Circ. & D. Ap. Resp. & Neoplasias & Endócrinas & D. Ap. Dig. \\
& $43,4 \%$ & $20,1 \%$ & $14,4 \%$ & $4,5 \%$ & $4,3 \%$ \\
Total & D. Ap. Circ. & Externas & Neoplasias & D. Ap. Resp. & D. Ap. Dig. \\
& $28,7 \%$ & $21,0 \%$ & $13,9 \%$ & $10,3 \%$ & $6,0 \%$ \\
\hline
\end{tabular}

*OMS/1995b

Fonte: www.datasus.gov.br (dados brutos)

Tabela 4

Número de óbitos, coeficientes de mortalidade ${ }^{\star}$ e razões entre os mesmos segundo causa

(Capítulos da CID-10) e sexo. Brasil, 2001.

\begin{tabular}{lrrrrc}
\hline \multirow{2}{*}{ Causa } & \multicolumn{2}{c}{ Masculino } & \multicolumn{2}{c}{ Feminino } & Razão M/F \\
& No & Coef. & No & Coef. & Coef. \\
\hline Doenças infecciosas e parasitárias & 27.254 & 32,1 & 17.713 & 20,8 & 1,5 \\
Neoplasias & 67.429 & 79,5 & 57.828 & 67,8 & 1,2 \\
Doenças do sangue e órgãos hematopoéticos & 2.713 & 3,2 & 2.523 & 3,0 & 1,1 \\
Doenças endócrinas, nutricionais & & & & & \\
e metabólicas & 21.203 & 25,0 & 26.541 & 31,1 & 0,8 \\
Transtornos mentais e comportamentais & 5.366 & 6,0 & 1.284 & 1,5 & 4,2 \\
Doenças do sistema nervoso & 6.648 & 7,8 & 5.636 & 6,6 & 1,2 \\
Doenças do olho e anexos & 9 & 0,0 & 3 & 0,0 & 1,0 \\
Doenças do ouvido & 70 & 0,1 & 59 & 0,1 & 1,0 \\
Doenças do aparelho circulatório & 139.216 & 164,1 & 123.956 & 145,4 & 1,1 \\
Doenças do aparelho respiratório & 49.994 & 58,9 & 40.212 & 47,2 & 1,2 \\
Doenças do aparelho digestivo & 29.175 & 34,4 & 15.173 & 17,8 & 1,9 \\
Doenças da pele e tecido subcutâneo & 793 & 0,9 & 1.030 & 1,2 & 0,8 \\
Doenças do sistema osteomuscular & 960 & 1,1 & 1.644 & 1,9 & 0,6 \\
Doenças do aparelho geniturinário & 7.691 & 9,1 & 6.644 & 7,8 & 1,2 \\
Gravidez, parto e puerpério & - & - & 1.583 & 1,9 & \\
Afecções do período perinatal & 19.207 & 22,6 & 14.733 & 17,3 & 1,3 \\
Malformações congênitas & 4.953 & 5,8 & 4.501 & 5,3 & 1,1 \\
Causas mal definidas & 75.762 & $\star *$ & 59.641 & $* *$ & $1,3 * * *$ \\
Causas externas & 102.200 & 120,4 & 18.521 & 21,7 & 5,5 \\
Total & 560.643 & 660,7 & 399.225 & 456,1 & 1,4 \\
& & & & &
\end{tabular}

* Por cem mil habitantes

**Não foram calculados

*** Razão entre os números absolutos de óbito

Fonte: www.datasus.gov.br (dados brutos) 
renti, 2003), realizado em sete cidades da América Latina, mediu a prevalência de oito condições ou doenças crônicas, baseando-se na resposta à pergunta sobre o fato de médico ou outro profissional de saúde ter dito ao entrevistado se o mesmo tinha determinada doença. $\mathrm{O}$ inquérito foi feito por meio de entrevistas domiciliárias para adultos de ambos os sexos com 60 anos e mais.

No município de São Paulo, para as oito condições inqueridas, verificou-se que a maior prevalência feminina ocorreu para cinco delas e, para os homens, para três (problema cardíaco, doença pulmonar obstrutiva crônica e embolia/derrame) (Tabela 5).

A prevalência de doenças crônicas aumenta com a idade e, particularmente, nos idosos, pode levar, às vezes, a uma grande limitação das atividades. Medir a limitação de atividades é uma maneira de avaliar as condições de saúde bem como os gastos que representam. Alguns países, como os Estados Unidos, vêm medindo esses agravos à saúde por meio do National Health Interview Survey, analisando as limitações com o auto-cuidado, com as atividades da vida diária, bem como aquelas impeditivas de ter um emprego fora de casa, e outras (NCHS, 2002). Para os idosos de 65 anos e mais, a limitação das atividades da vida diária, conseqüente a doenças crônicas, nos Estados Unidos, foi sempre menor nos homens (5,1\% e, nas mulheres, $7 \%$, em 2000).

Diferentemente da mortalidade, para a qual se tem uma grande tradição na apresentação de dados segundo numerosas variáveis, com a morbidade isso é muito mais recente e se destacam as estatísticas referentes à internação hospitalar, nas quais se pode verificar aspectos específicos para os sexos, particularmente, quanto às causas.

\section{Análise das internações hospitalares}

Do ponto de vista de análise da morbidade, é preciso comentar que se referem aos casos mais graves e que necessitaram de internação.

Nos Estados Unidos, verifica-se que as altas femininas são em número maior que as dos homens ( $53,1 \%$ e $46,9 \%$, respectivamente) (Graves, 1993), sendo que, em parte, isto poderia ser explicado pelas internações para parto; entretanto, esse excesso de altas não se deve apenas a esse fato, mas à maior freqüência das internações por vários outros agravos. Medidos em termos de coeficientes, os valores são 129,8 e 99,9 por mil habitantes do sexo, respectivamente. Por outro lado, a duração média (em dias de internação) é maior no sexo masculino (5,4 e 4,6 dias de internação para homens e mulheres). Uma possível hipótese para tal fato é que, talvez, os homens se internariam em uma fase mais avançada da doença, o que prolongaria o tratamento. Isto, se verdadeiro, indicaria uma questão comportamental, isto é, ligada ao gênero.

A situação no Brasil, por meio da análise das saídas em hospitais próprios ou conveniados com o SUS em 2002, mostra que ocorre também um excesso de saídas no sexo feminino (cerca de 60\% a mais). Excluídas as internações ligadas a problemas de gravidez, parto e puerpério (2.731.766), nota-se um equilíbrio entre os dois sexos (Tabela 6).

Quanto à distribuição segundo causas, a razão entre hospitalizações masculinas e femininas está balanceada, com exceção das interna-

Tabela 5

Respostas positivas (\%) à pergunta sobre doenças/condições crônicas segundo idade (em anos) e sexo. Projeto SABE, Município de São Paulo, 2002.

\begin{tabular}{lcccccc}
\hline Doença/Condição & \multicolumn{2}{c}{$\mathbf{6 0} \mathbf{~ a 4}$} & \multicolumn{2}{c}{$\mathbf{7 5}$ e mais } & \multicolumn{2}{c}{ Total } \\
& Masc. & Fem. & Masc. & Fem. & Masc. & Fem. \\
\hline Pressão alta & 49,8 & 55,1 & 45,9 & 60,2 & 49,1 & 56,3 \\
Artrite/Artrose/Reumatismo & 20,0 & 38,5 & 23,2 & 43,3 & 20,6 & 39,6 \\
Problema cardíaco & 19,5 & 17,2 & 24,7 & 24,0 & 20,5 & 18,8 \\
Diabetes & 17,2 & 19,3 & 14,9 & 17,0 & 16,8 & 18,7 \\
Osteoporose & 2,4 & 21,8 & 4,3 & 23,8 & 2,7 & 22,3 \\
Doença pulmonar obstrutiva Crônica & 13,8 & 10,7 & 16,4 & 10,7 & 14,3 & 10,7 \\
Embolia / Derrame & 8,6 & 5,7 & 10,5 & 6,9 & 8,9 & 6,0 \\
Tumor maligno & 2,9 & 3,0 & 4,0 & 5,0 & 3,1 & 3,5 \\
\hline
\end{tabular}

Fonte: Lebrão, Laurenti 2003 
Tabela 6

Número e proporção (\%) de internações e razão entre as mesmas, segundo sexo e causa (Capítulos CID-10). Brasil, 2002.

\begin{tabular}{|c|c|c|c|c|c|}
\hline \multirow[t]{2}{*}{ Causa } & \multicolumn{2}{|c|}{ Masculino } & \multicolumn{2}{|c|}{ Feminino } & \multirow[t]{2}{*}{ Razão M/F } \\
\hline & № & $\%$ & № & $\%^{*}$ & \\
\hline Doenças infecciosas e parasitárias & 534.834 & 11,8 & 507.295 & 11,4 & 1,05 \\
\hline Neoplasias & 205.914 & 4,6 & 321.829 & 7,2 & 0,64 \\
\hline Doenças do sangue e órgãos hematopoéticos & 34.063 & 0,8 & 43.730 & 1,0 & 0,78 \\
\hline Doenças endócrinas, nutricionais e metabólicas & 144.019 & 3,2 & 178.074 & 4,0 & 0,81 \\
\hline Transtornos mentais e comportamentais & 201.827 & 4,5 & 106.399 & 2,4 & 1,90 \\
\hline Doenças do sistema nervoso & 87.458 & 1,9 & 70.872 & 1,6 & 1,23 \\
\hline Doenças do olho e anexos & 29.832 & 0,7 & 31.102 & 0,7 & 0,96 \\
\hline Doenças do ouvido & 8.166 & 0,2 & 8.180 & 0,2 & 1,00 \\
\hline Doenças do aparelho circulatório & 563.543 & 12,5 & 652.846 & 14,7 & 0,86 \\
\hline Doenças do aparelho respiratório & 954.828 & 21,1 & 865.796 & 19,4 & 1,10 \\
\hline Doenças do aparelho digestivo & 528.126 & 11,7 & 458.277 & 10,3 & 1,15 \\
\hline Doenças da pele e tecido subcutâneo & 69.728 & 1,5 & 55.386 & 1,2 & 1,26 \\
\hline Doenças do sistema osteomuscular & 153.806 & 3,4 & 122.712 & 2,8 & 1,25 \\
\hline Doenças do aparelho geniturinário & 234.335 & 5,2 & 536.014 & 12,0 & 0,44 \\
\hline Gravidez, parto e puerpério & - & - & 2.731 .766 & * & * \\
\hline Afecções do período perinatal & 105.744 & 2,3 & 95.736 & 2,1 & 1,10 \\
\hline Malformações congênitas & 48.324 & 1,1 & 40.441 & 0,9 & 1,19 \\
\hline Causas mal definidas & 63.072 & 1,4 & 79.087 & 1,8 & 0,80 \\
\hline Lesões & 495.644 & 11,0 & 213.182 & 4,8 & 2,32 \\
\hline Contatos com serviços & 64.236 & 1,4 & 67.450 & 1,5 & 0,95 \\
\hline Total & 4.527 .499 & 100,0 & 7.186 .174 & 100,0 & 0,63 \\
\hline
\end{tabular}

* Proporção calculada com exclusão das causas ligadas a gravidez, parto e puerpério

Fonte: www.datasus.gov.br (dados brutos)

ções por lesões e envenenamentos $(2,32: 1)$ e transtornos mentais e comportamentais (1,90:1). A menor proporção de internações masculinas diante das femininas ocorre entre as relativas a doenças do aparelho geniturinário (razão igual a $0,44: 1)$ e a neoplasias $(0,64: 1)$.

Analisando, no sexo masculino, as principais causas que levam à internação, aparecem em primeiro lugar as doenças do aparelho respiratório $(21,1 \%)$, seguindo-se, com proporções semelhantes, doenças do aparelho circulatório, infecciosas, aparelho digestivo e lesões.

Nas idades, detectam-se diferenças importantes. Assim, nos menores de 10 anos as hospitalizações concentram-se nas causas respiratórias $(39,5 \%)$, seguindo-se as infecciosas $(24,1 \%)$. Nos adolescentes e adultos jovens, verifica-se maior freqüência no atendimento de lesões (mais de $20 \%$ ). Na faixa de 20 a 39 anos, chama a atenção a presença de transtornos mentais (11,3\%). Nas idades de 40 anos e mais, com proporções crescentes segundo as idades, surgem as doenças do aparelho circulatório, alternando posições, as doenças dos aparelhos respiratório e digestivo (Tabela 7).
Quando se comparam as internações (Tabela 7) e a mortalidade (Tabela 4) por grandes grupos de causas no sexo masculino, verificamse diferenças. A mortalidade por doenças cardiovasculares aparece em primeiro lugar, enquanto nas internações, o primeiro posto é ocupado pelas doenças do aparelho respiratório, com proporção equivalente ao dobro daquela por doenças cardiovasculares e mais de quatro vezes a das neoplasias. É interessante verificar que os transtornos mentais são causas importantes de internações, com um número de casos um pouco menor do que as neoplasias, em 2002, para o País. Também as conseqüências das causas externas corresponderam a $100 \%$ a mais do que as neoplasias. Quanto a essas últimas, as internações por câncer de próstata se apresentam como uma das causas mais importantes.

\section{Alguns agravos importantes na saúde do homem}

Ao serem abordados aspectos da saúde masculina, as atuações da síndrome da imunodefi- 
Tabela 7

Principais causas de internação no sexo masculino segundo grupo etário. Brasil, 2002.

\begin{tabular}{|c|c|c|c|c|c|}
\hline Idade (em anos) & 1o Posto & 2o Posto & 3o Posto & 4o Posto & 5o Posto \\
\hline 0 a 9 & $\begin{array}{l}\text { D. Ap. Resp. } \\
39,5 \%\end{array}$ & $\begin{array}{c}\text { Infecciosas } \\
24,1 \%\end{array}$ & $\begin{array}{c}\text { Perinatais } \\
9,0 \%\end{array}$ & $\begin{array}{c}\text { D. Ap. Digest. } \\
7,3 \%\end{array}$ & $\begin{array}{c}\text { Lesões } \\
5,5 \%\end{array}$ \\
\hline 10 a 19 & $\begin{array}{l}\text { Lesões } \\
23,60 \%\end{array}$ & $\begin{array}{l}\text { D. Ap. Resp. } \\
17,80 \%\end{array}$ & $\begin{array}{c}\text { Infecciosas } \\
13,00 \%\end{array}$ & $\begin{array}{c}\text { D. Ap. Digest. } \\
10,60 \%\end{array}$ & $\begin{array}{c}\text { Ap. Geniturinário } \\
5,70 \%\end{array}$ \\
\hline 20 a 39 & $\begin{array}{l}\text { Lesões } \\
21,3 \%\end{array}$ & $\begin{array}{c}\text { D. Ap. Digest. } \\
14,0 \%\end{array}$ & $\begin{array}{c}\text { Transt. Mentais } \\
11,3 \%\end{array}$ & $\begin{array}{c}\text { Infecciosas } \\
10,6 \%\end{array}$ & $\begin{array}{l}\text { D. Ap. Resp. } \\
10,2 \%\end{array}$ \\
\hline 40 a 59 & $\begin{array}{c}\text { D. Ap. Circ. } \\
18,6 \%\end{array}$ & $\begin{array}{c}\text { D. Ap. Digest. } \\
16,1 \%\end{array}$ & $\begin{array}{c}\text { D. Ap. Resp. } \\
11,7 \%\end{array}$ & $\begin{array}{l}\text { Lesões } \\
10,2 \%\end{array}$ & $\begin{array}{c}\text { Infecciosas } \\
7,9 \%\end{array}$ \\
\hline 60 a 79 & $\begin{array}{l}\text { D. Ap. Circ. } \\
29,2 \%\end{array}$ & $\begin{array}{l}\text { D. Ap. Resp. } \\
19,0 \%\end{array}$ & $\begin{array}{c}\text { D. Ap. Digest. } \\
11,5 \%\end{array}$ & $\begin{array}{c}\text { Neoplasias } \\
7,9 \%\end{array}$ & $\begin{array}{c}\text { Ap. Geniturinário } \\
6,7 \%\end{array}$ \\
\hline 80 e mais & $\begin{array}{l}\text { D. Ap. Circ. } \\
30,5 \%\end{array}$ & $\begin{array}{l}\text { D. Ap. Resp. } \\
25,1 \%\end{array}$ & $\begin{array}{c}\text { D. Ap. Digest. } \\
7,7 \%\end{array}$ & $\begin{array}{c}\text { Infecciosas } \\
7,5 \%\end{array}$ & $\begin{array}{c}\text { Ap. Geniturinário } \\
6,3 \%\end{array}$ \\
\hline Total & $\begin{array}{l}\text { D. Ap. Resp. } \\
21,1 \%\end{array}$ & $\begin{array}{l}\text { D. Ap. Circ. } \\
12,4 \%\end{array}$ & $\begin{array}{c}\text { Infecciosas } \\
\quad 11,8 \%\end{array}$ & $\begin{array}{c}\text { D. Ap. Digest. } \\
11,7 \%\end{array}$ & $\begin{array}{l}\text { Lesões } \\
10,9 \%\end{array}$ \\
\hline
\end{tabular}

Fonte: www.datasus.gov.br (dados brutos)

ciência adquirida (Aids) e do alcoolismo necessitam ser comentadas.

A Aids, desde o seu início, sempre se associou ao sexo masculino e com determinados comportamentos de risco. Este foi, por mais contraditório que pareça, um dos fatores que ajudou sua propagação, uma vez que as atividades preventivas foram direcionadas para grupos específicos que apresentavam comportamento de maior risco, deixando, a descoberto, outros setores da sociedade (Laurenti et al., 1998).

O padrão epidemiológico da Aids vem se alterando e, à medida que diminui a proporção de casos entre homossexuais e bissexuais, aumenta o número de indivíduos heterossexuais, o que mantém elevado o coeficiente de incidência no sexo masculino e propicia aumento de incidência no sexo feminino. A análise da transmissão do HIV, entre usuários de drogas, não encontra respaldo nas questões de gênero. $\mathrm{O}$ risco da contaminação é uniforme entre usuários, na dependência, apenas, do compartilhamento de seringa e agulhas; no entanto, a droga-adição é mais freqüente entre os homens.

Outro aspecto marcante na saúde masculina é o alcoolismo em suas diversas manifestações. A Organização Pan-Americana de Saúde reconheceu que o uso de álcool, assim como de outras substâncias psicoativas, cresceu de maneira significante nos países da América entre 1970 e 1980 (OPS, 1990b).

A freqüência do alcoolismo não é, ainda, bem conhecida existindo uma certa carência de estudos sobre o tema e os existentes referem-se a áreas restritas, sendo que seus resultados não podem ser generalizados.

Um indicador importante para se avaliar o problema é a medida do efeito do alcoolismo sobre a saúde, visto por meio da freqüência da psicose alcoólica e da síndrome da dependência do álcool, dentre os transtornos mentais, e da cirrose hepática, dentre as doenças do aparelho digestivo. Tem sido usual avaliar este impacto do uso por meio da mortalidade e da morbidade hospitalar. Comparado com outros diagnósticos, mostra que somente as violências apresentam diferenciais entre os sexos mais elevados, quer os de mortalidade, quer os de morbidade.

Prevenir o alcoolismo, e com isso suas graves conseqüências, é um desafio, particularmente, em regiões mais pobres, onde o uso excessivo de bebidas alcoólicas parece fazer parte do comportamento masculino. Para programas que visem reduzir o alcoolismo, o conhecimento do problema deve englobar aspectos culturais e comportamentais que se associam ao agravo, donde a necessidade de se avaliar a questão sob enfoque de gênero.

$\mathrm{O}$ aspecto comportamental influi significantemente na saúde humana, sendo que a expressão estilo de vida vem sendo cada vez mais utilizada, e o estilo do homem, sob várias óticas, se diferencia daquele da mulher.

A estratégia de prevenção e promoção da saúde tem de levar em conta a mudança compor- 
tamental, em toda a população, tendo em mente as diferenças de gênero em relação ao hábito de fumar, ao alcoolismo, ao tipo de dieta, ao ambiente de trabalho, à atividade física, ao peso corporal, entre outros. Fica bastante claro que a presença de muitas doenças que afetam a população, muitas vezes mais acentuadamente a masculina, tem mecanismos bastante conhecidos e aceitos cientificamente; o difícil, muitas vezes, é como incorporá-los à prática diária.

\section{Colaboradores}

R Larenti, MHP Mello Jorge e SLD Gotlieb participaram igualmente de todas as etapas da elaboração do artigo.

\section{Nota dos autores}

Em 1997, o prof. dr. João Yunes, então diretor da Divisão de Promoção e Proteção da Saúde da Organização Pan-Americana da Saúde - Washington, sensível ao problema de que pouco ou quase nada se discutia sobre a saúde do homemcontrariamente à da mulher, bastante analisada -, solicitou estudo específico sobre o tema. Originou-se daí o trabalho Perfil epidemiológico da saúde masculina na Região das Américas: uma contribuição para o enfoque de gênero, no qual este artigo se fundamenta. Os autores, com a atual publicação, homenageiam o professor Yunes e agradecem a colaboração dos alunos Bruno Zoca de Oliveira, mestrando da Faculdade de Saúde Pública da USP; e Vanessa Luiza Tuono, da Escola de Enfermagem da USP, bolsista de Iniciação Científica do CNPq.

\section{Referências bibliográficas}

Adams PF \& Benson V 1990. Current estimates from the National Health Interview Survey, 1989. National Center for Health Statistics. Vital Health Statistics 10(176).

Bobadilha JL, Frenk J, Lozano R, Frejka T \& Stern C 1993. The epidemiological transition and health priorities, pp. 161-187. In DT Janison. Disease control priorities in developing countries. Oxford Medical Publications: Oxford University Press, Oxford.

Cesar CLG et al., 1991. Morbidade referida e utilização de serviços de saúde na Região Sudoeste da Grande São Paulo, 1989-1990: Relatório Final. Faculdade de Saúde Pública da USP. São Paulo.

Graves EV 1993. National Hospital Discharge Survey: annual summary. Hyattsville, MD. National Center for Health Statistics. Series 13, no 106.

Hartigan P 1998. Women and health for Beijing agenda. Meeting of the Canadian Society for International Health. Canadá.

Laurenti R 1990. Transição demográfica e transição epidemiológica, pp. 143-165. In Anais do Io Congresso Brasileiro de Epidemiologia. Campinas.

Laurenti R, Buchalla CM, Mello Jorge MHP, Lebrão ML \& Gotlieb SLD 1998. Perfil epidemiológico da saúde masculina na Região das Américas: uma contribuição para o enfoque de gênero. Centro Colaborador da OMS para a Classificação de Doenças em Português, São Paulo. 
Lebrão ML \& Laurenti R 2003. Condições de saúde. In ML Lebrão \& YA Oliveira Duarte (orgs.). SABE Saúde, Bem-estar e Envelhecimento. O Projeto SABE no Município de São Paulo: Uma abordagem inicial. Organização Pan-Americana da Saúde, Brasília.

MacMahon B \& Pugh TF 1970. Epidemiology: principles and methods. Little, Brown and Company, Boston.

Madigan FC 1957. Are sex mortality differentials biologically caused? The Millbank Memorial Fund Quarterly, 35:203-223.

Murray JLC, Yang G \& Qiao X 1992. Adult mortality: levels, patterns and causes. In The health of adults in developing world. Oxford University Press. Nova York.

National Center For Health Statistics (NCHS) 1993. Health people 2000. Review Health United States 1992. Hyattsville, Maryland, Public Health Service.

National Center For Health Statistics 2002 (NCHS). Dept. of Health and Human Services - Health. United States, 2002. Hyattsville, Maryland.

OMS 1995a. Epidemiologia y prevención de las enfermedades cardiovasculares en los ancianos. (Série de Informes Técnicos 853). Genebra.

OMS 1995b. Classificação estatística internacional de doenças e problemas relacionados à saúde - 10a Revisão. Centro Brasileiro de Classificação de Doenças, Edusp. São Paulo.

OPS 1990a. Perfil epidemiológico de la salud de la mujer en la Región de las Américas. Washington, DC.
OPS 1990b. Las condiciones de salud en las Américas. Washington, DC (OPS - Publicación Científica 524).

OPS 1993. Genero, mujer y salud en las Américas. Washington, DC (OPS - Publicación Científica 541).

OPS 2003. Situación de la salud en las Américas. Indicadores e dados básicos. (OPS/AIS/03.01).

Rede Interagencial de Informações para a Saúde (Ripsa) 2004. Indicadores e Dados Básicos - IDB - 2003. Brasília (folder).

Rothman KJ 1996. Lessons from John Graunt. Lancet (347):37-39.

Torrey BB, Kiurella K \& Taeuber CM 1987. An aging world. Washington, DC US Government Printing Office (US Bureau of the Census, International Population Reports).

Van Nostrand JF, Furner SE \& Suzman R (eds.) 1993. Health data on older Americans: United States, 1992. Hyattsville, Maryland, National Center for Health Statistics. Vital and Health Statistics, Series 3, no 27.

Verbrugge L 1989. Recent, present and future health of American adults. American Review of Public Health 10:333-361.

Waldman EA 2000. A transição epidemiológica: tendências e diferenciais dos padrões de morbimortalidade em diferentes regiões do mundo. O Mundo da Saúde 24(24):10-18. Disponível em <www.datasus.gov.br>.

Artigo apresentado em 13/8/2004

Aprovado em 13/9/2004

Versão final apresentada em 24/8/2004 